\title{
LAS LEYES DE LICENCIA DE MATERNIDAD Y EL MERCADO LABORAL EN COLOMBIA
}

\author{
HÉCTOR ALBERTO BOTELLO-PEÑALOZA \\ ISAAC GUERRERO-RINCÓN*
}

\section{RESUMEN}

Este trabajo analiza el impacto de la Ley 1822 de 2017, que aumentó la licencia de maternidad de 14 a 18 semanas, en el mercado laboral femenino colombiano. Para evaluar esta política, la investigación estima las tasas en torno a la probabilidad de ser inactivo o informal en tres cohortes de población diferentes: las mujeres de 15 a 30 años, las mujeres de 31 a 65 años y los hombres de 15 a 65 años. Se encuentra que la ampliación de la licencia de maternidad aumenta la probabilidad de que las mujeres con altas tasas de fecundidad sean informales e inactivas.

Palabras clave: Colombia, licencia de maternidad, mercado laboral, regulación laboral, licencia de paternidad.

Clasificaciones JEL: J08, J2, J3, J7, K31

\footnotetext{
* Hector Alberto Botello-Peñaloza es miembro del Grupo de Investigacion sobre Desarrollo Regional y Planeación Espacial, Universidad Nacional de Colombia, Bogotá; Isaac Guerrero-Rincón es Profesor y miembro del Grupo de Investigación sobre Desarrollo Regional y Planeación Territorial, Universidad Industrial de Santander, Bucaramanga. Correos electrónicos: hbotellop@unal.edu.co.y iguerrin@uis.edu.co. Este trabajo hace parte del proyecto Capital Social y Desarrollo, activo desde 2013. Recibido: 12 de abril de 2019; aceptado: 4 de junio de 2019.
}

Economía \& Región, Vol. 13, No. 1, (Cartagena, junio 2019), pp. 67-86. 


\section{ABSTRACT \\ Maternity leave laws and the labor market in Colombia}

We analyze the impact of Law 1822 of 2017, which increased maternity leave from 14 to 18 weeks, on the Colombian female labor market. To evaluate this policy, we estimate the probability of being inactive or informal in three different population cohorts: women aged 15 to 30 , women aged 31 to 65 and men aged 15 to 65 . We find that extending maternity leave increases the likelihood that women with high fertility rates will be informal and inactive.

Key words: Colombia, maternity leave, labor market, labor regulation, paternity leave.

JEL Classifications: J08, J2, J3, J7, K31

\section{INTRODUCCIÓN}

Las mujeres de todo el mundo han encontrado más oportunidades de empleo en la economía, tanto en países emergentes y desarrollados. Este fenómeno se observa en el aumento de los indicadores de participación laboral y la importancia de los ingresos que las mujeres proveen dentro de los hogares. Una mayor integración ha extendido la representación de las mujeres, mostrando su potencial en las cadenas productivas, (Lagarde, 2014). Sin embargo, hoy en día en los países en desarrollo menos de la mitad de todas las mujeres hacen parte del mercado laboral y sufren de discriminación salarial asociada únicamente a su condición de género o de madres (Botello, 2017; Botello y Alba, 2014).

Estos fenómenos excluyentes pueden llevar a una pérdida de competitividad de las economías. Lagarde (2014) considera que, si la participación laboral de las mujeres pudiera ser igual a la de los hombres, el PIB aumentaría 5\% en Estados Unidos, 9\% en Japón, 12\% en los Emiratos Árabes Unidos y 34\% en Egipto. Para proteger la condición de la mujer, la legislación laboral de muchos países incluye un conjunto de políticas orientadas a otorgar una vacante remunerada durante los primeros meses de atención por dar a luz, lo que se conoce como "baja por maternidad" (Botello, 2015).

Según varios estudios, la licencia de maternidad tiene muchos beneficios para la madre y el niño. Por ejemplo, la lactancia materna reduce la propensión de los 
lactantes a sufrir algunas enfermedades gástricas o respiratorias e infecciosas (OMS, 2003; Martin et. al., 2004). Para la madre lactante, las tasas de cáncer de mama y de ovario son más bajas, y la diabetes y los problemas cardíacos son menores. (Victora et. al., 2003).

De otra parte, se ha encontrado evidencia empírica de que la licencia de maternidad aumenta los costos laborales para las empresas, reduciendo los incentivos a contratar mujeres en favor de hombres (Ramírez et. al., 2015). Esto ocurre incluso si el permiso de maternidad se paga con cargo a la seguridad social porque hay otros costos no laborales asociados a pérdidas de productividad debidas a la sustitución, nuevos costos de formación, entre otros (Forero de Saade et. al., 1991). Para Hyclak et. al. (2012, p. 384), en un escenario donde madres y no madres ubicados en la misma ocupación y la misma productividad, existe una penalización salarial dado que los empleadores supondrán que el cuidado de niños y las tareas domésticas afectarán el desempeño en la empresa, lo que hace que piensen subjetivamente que las madres son menos productivas y les asignen menores salarios. Estos fenómenos pueden causar una menor inversión en capital humano por parte de las madres, lo que llevaría a un crecimiento salarial más lento (Livermore et. al., 2011, p. 19). En esta misma línea Eagly y Carli (2007) mencionan que los individuos hacen prejuicios conscientes e inconscientes sobre los hombres, y las mujeres. El mismo comportamiento en un hombre y en una mujer se califica de distinta manera. En este sentido, el matrimonio y la paternidad están asociados a mayores salarios para los hombres, pero no para las mujeres

Sin embargo, autores como Oaxaca (2007) afirman que medir la discriminación en el mercado laboral es una tarea ardua por la multitud de factores por el lado de la oferta y demanda laboral. En consecuencia, ciertos estudios van en contra de la discriminación y la adjudican a otros fenómenos. Por ejemplo, Goldin (2014, p. 226) concluye que la brecha de género en los salarios no es por discriminación sino por la composición de los empleos en los que se embarcan las mujeres, como los puestos directivos. De Cabo y Rodríguez (2014, p. 13) afirman que son resultado de la distribución de las horas que trabajan mujeres y hombres.

Esta investigación quiere contribuir a estimar los efectos generados por el aumento de la licencia de maternidad en la Ley 1822 de 2017 en Colombia de las mujeres trabajadoras mediante sus tasas de actividad e informalidad. Los datos se tomaron de las encuestas de hogares colombianos entre enero de 2015 y septiembre de 2017. Se emplea un modelo probabilístico con variables dicotómicas y socioeconómicas. Para mejorar la estimación, se estudian tres grupos: los hombres 
y dos grupos de población femeninos según sus tasas de fecundidad: mujeres de alta fecundidad (18-35 años) y de baja fecundidad (36 años o más). Los resultados muestran que la extensión de la licencia de maternidad en Colombia aumenta las tasas informales y de inactividad de las mujeres entre 15 y 30 años. En promedio, la participación laboral se reduce en un $0,7 \%$ y la probabilidad de ser informal aumenta en un $0,57 \%$. Estos resultados son consistentes con lo que se observa en la literatura internacional.

El documento se divide en cinco partes. En la segunda sección se presenta la situación de la licencia de maternidad en Colombia y la literatura relacionada con sus consecuencias en el mercado laboral femenino. En la tercera se describen los datos y el modelo econométrico utilizado en el estudio. Por último, en la cuarta y quinta secciones se presentan los resultados, las recomendaciones de política y las conclusiones.

\section{LAS LEYES DE MATERNIDAD EN COLOMBIA}

Según Addati et. al. (2014), todos los países del mundo han establecido leyes de maternidad para la protección de las nuevas madres. La Organización Internacional del Trabajo (OIT) en el año 2000 se reunió en Ginebra para crear la recomendación C183 - Convenio sobre la protección de la maternidad, un documento de 18 puntos que trazaba lineamientos a sus países miembros para continuar y mejorar el estado de protección de la mujer. Dentro de estos se planteaba la creación de sistemas de mitigación del riesgo para la población de madres en gestación y lactantes basadas en un sistema de prestaciones sociales como la licencia de maternidad. Esta se extendía a 18 semanas de ausencia remunerada, con un pago sobre la licencia no inferior a dos tercios del salario medio devengado, financiable a través de la seguridad social o los fondos públicos destinados para este fin.

Según los propios datos de la OIT, 34\% de 185 países subscriptos cumplen plenamente la recomendación sobre las leyes de maternidad de la C183. Sin embargo, existe una gran diversidad en el alcance de estas leyes en todo el mundo. Según Addati et. al. (2014) se pueden clasificar los siguientes grupos de países:

- 53\% (98 países) posee una norma de al menos 14 semanas de licencia.

- 42 países cumplen o superan el permiso propuesto de 18 semanas. 
- $60 \%$ de los países legisla con una licencia de entre 12 y 13 semanas, menor que la prevista en el último Convenio (C183), pero en consonancia con los convenios anteriores (que datan de 1952).

- Sólo el 15\% de los territorios conceden menos de 12 semanas.

A menos que se trate de convenios internacionales, sólo el 34,4\% de los países tienen todos los derechos a una vacante remunerada. La condición más crítica se presenta en regiones con alta discriminación y pobreza de las mujeres, por ejemplo, Oriente Medio y África. En América Latina, la mitad de los territorios siguen las normas de la orT, pero la informalidad y la desigualdad de ingresos siguen siendo problemas graves que dificultan el pleno cumplimiento de las convenciones internacionales. El Cuadro 1 muestra las leyes de maternidad en América Latina mediante cuatro características claves: duración, valor, fuente y tipo de financiamiento.

\section{CUADRO 1}

Características de las leyes de maternidad en América Latina

\begin{tabular}{|c|c|c|c|c|}
\hline País & Duración & Valor de la licencia (\% del salario) & Fuente & $\begin{array}{c}\text { Tipo de } \\
\text { financiación }\end{array}$ \\
\hline Argentina & 90 días & $100 \%$ & Contribuciones a la seguridad social & Obligatorio \\
\hline Bolivia & 60 días & $\begin{array}{l}\text { Si es un salario mínimo es del } \\
100 \% \text {. En otro caso, } 70 \% \text { de las } \\
\text { diferencias entre salario medio y } \\
\text { salario mínimo. }\end{array}$ & Contribuciones a la seguridad social & Obligatorio \\
\hline Brasil & 16 semanas & $100 \%$ & Contribuciones a la seguridad social & Obligatorio \\
\hline Chile & 18 semanas & $100 \%$ & Contribuciones a la seguridad social & Obligatorio \\
\hline Colombia & 14 semanas & $100 \%$ & Contribuciones a la seguridad social & Obligatorio \\
\hline Ecuador & 12 semanas & $100 \%$ & $\begin{array}{l}\text { Mixto ( } 75 \% \text { seguridad social; } 25 \% \\
\text { empleador) }\end{array}$ & Obligatorio \\
\hline México & 12 semanas & $100 \%$ & Contribuciones a la seguridad social & Obligatorio \\
\hline Nicaragua & 12 semanas & $100 \%$ & $\begin{array}{l}\text { Mixto (60\% seguridad social; } 40 \% \\
\text { empleador }\end{array}$ & Obligatorio \\
\hline Panamá & 14 semanas & $100 \%$ & Mixto & \\
\hline Paraguay & 12 semanas & $50 \%$ por 9 semanas & Contribuciones a la seguridad social & Obligatorio \\
\hline Perú & 90 días & $100 \%$ & Contribuciones a la seguridad social & Obligatorio \\
\hline Uruguay & 12 semanas & $100 \%$ & Contribuciones a la seguridad social & Obligatorio \\
\hline Venezuela & 18 semanas & $100 \%$ & Contribuciones a la seguridad social & Obligatorio \\
\hline
\end{tabular}

Fuente: OIT (2010). Maternidad y Trabajo. 
En cuanto a la licencia de paternidad, en Chile y Brasil la ley establece cinco días de vacante remunerada, en Perú cuatro y en Argentina dos días. En México y Panamá no existe ninguna ley de paternidad.

Antes de 1975, Colombia tenía un sistema de seguridad social donde la licencia de maternidad era pagada únicamente por el empleador. Sin embargo, a partir de ese año los costos directos fueron compartidos con el empleado a partir de las cotizaciones realizadas por ambos al sistema de seguridad social. Antes de la Ley 1822 de 2017, las mujeres tenían 14 semanas de licencia de maternidad, y recibían el $100 \%$ del salario que ganaban. En términos de financiación, el empleador debe cubrir el 73,06\% de su salario para ese año (el resto del salario está cubierto por el sistema de seguridad social), el 30,31\% corresponde al salario de la trabajadora sustituta y el 3,37\% al valor de una hora pagada por día de lactancia materna, durante los tres meses posteriores al regreso del permiso de maternidad. Sobre el costo de la licencia de maternidad para las empresas colombianas, las estimaciones de Según Espino y Salvador (2014) el costo de la licencia de maternidad para las empresas colombianas es de 6,73\% del salario.

Sin embargo, la nueva Ley modifica el artículo 236 del Código Sustantivo del Trabajo, aumentando la licencia de maternidad en cuatro semanas, un incremento del $28 \%$, que se contabiliza a partir del día del parto o del tiempo estipulado por el médico que la madre debe ausentarse del trabajo. Si la madre trabaja por horas o por deberes, se tendrá en cuenta el salario medio percibido en el último año de servicio. Si el parto es múltiple, el permiso será de 20 semanas. Sin embargo, la licencia de paternidad no se prorrogó y se mantuvo en ocho días laborables. Entre otros alcances de la reforma, se establece que las entidades públicas y las empresas privadas con activos iguales o superiores a 1500 salarios mínimos, o con capital por debajo de esa cantidad, pero que tengan más de 50 empleados, deben condicionar salas de maternidad especializadas para la lactancia. Sobre este punto, el Ministerio de Salud tuvo seis meses para establecer los criterios técnicos para el montaje de estas salas, con plazos de montaje para empresas públicas y privadas de dos a cinco años.

\section{REVISIÓN DE LA LITERATURA}

Estudios empíricos han encontrado una relación entre los costos laborales de la licencia de maternidad y el menor incentivo para que las empresas contraten mujeres, un efecto que puede ser considerable a largo plazo, tanto en países desarro- 
llados (Jaumotte, 2003; Bergemann y Riphahn 2010) como en países emergentes (Ramírez et. al., 2015). Pero la evidencia empírica arroja ciertos resultados contradictorios. Algunos estudios concluyen que la licencia de maternidad reduce los salarios de las mujeres (Schönberg y Ludsteck, 2007; Ruhm, 1998), pero también encuentran un aumento en los salarios (Rossin-Slater et. al., 2013), mientras que otro grupo no encuentra ningún efecto (Baum, 2003; Baker y Milligan, 2008; Hashimoto et. al., 2004). Por ejemplo, Blau y Kahn (2013) encuentran que el aumento de las leyes de familia, como la licencia de maternidad, es responsable del 28-29\% de la disminución de la participación de las mujeres estadounidenses en la fuerza laboral, en comparación con los hombres. En este trabajo se encontró que las diferentes metodologías de evaluación requieren la presencia de grupos de control no experimentales que no se vean afectados por la legislación de maternidad. Esta línea de pensamiento se reflejará en el resto de los trabajos analizados.

Lai y Masters (2005) evalúan el efecto de la introducción de la licencia obligatoria de maternidad en la participación laboral de las mujeres en Taiwán. Los autores infieren que la implementación de estas leyes reduce significativamente la probabilidad de empleo y también su salario. Gruber (1994) estudia los efectos en el mercado laboral de la legislación estadounidense entre 1975 y 1978, que obligó a las empresas a incluir los costos de maternidad y parto en los planes de salud de los empleados. El estudio reveló una disminución significativa de los salarios, pero no de los niveles de empleo. Schönberg y Ludsteck (2007) concluyen que la ampliación de la cobertura de licencias en el mercado laboral alemán indujo a las mujeres a retrasar su regreso al mercado de trabajo y redujo sus salarios. Sin embargo, este efecto es compensado por un efecto de selección positivo, resultado de un efecto global nulo o positivo, lo cual es consistente con la investigación de Baum (2003) y Baker y Milligan (2008).

Algunas investigaciones estudian si los empleadores discriminan a las mujeres porque se las considera menos competentes por el único efecto de la maternidad. Cuddy et. al. (2004) presentan evidencia de sesgo al describir cómo los evaluadores califican a una consultora como menos competente cuando se la describe como una mujer con hijos. Según Correll y Bernard (2007) esta situación se debe parcialmente al papel cultural atribuido a la maternidad, que está en conflicto con las creencias culturales asociadas con una trabajadora ideal. Los diseños metodológicos de estos estudios se basan en métodos experimentales directos. Cuando los participantes evaluaron los mismos currículos de un par de candidatas del mismo sexo cualificadas para el puesto de trabajo, lo único en que difieren es en su estado de maternidad. Por el contrario, los hombres se benefician de su condición 
de padres. Por ejemplo, se percibe que los padres están más comprometidos con su trabajo y se les ofrece un salario inicial significativamente más alto que a los hombres que no son padres.

En Colombia, la investigación económica entre 1970 y 1990 buscó determinar la influencia de los altos costos laborales causados por la licencia de maternidad en la participación laboral de las mujeres. Se llegó a la conclusión de que parte de la baja participación ocupacional femenina se debía al mayor costo derivado de la licencia de maternidad (Forero de Saade et. al., 1991). En un documento relacionado, Iragorri (2012) evaluó el efecto de una decisión judicial de la Corte Constitucional que establecía la invalidez del despido y exigía la reincorporación de todas las trabajadoras embarazadas dentro de los tres meses siguientes al parto. A partir de los datos de la Gran Encuesta Integrada de Hogares, se observa que la participación femenina en la fuerza de trabajo disminuyó especialmente en el caso de las mujeres de entre 15 y 29 años de edad.

Gutiérrez (2008) observó los salarios a nivel urbano y rural en la Encuesta Nacional de Calidad de Vida 2003. Sus estimaciones muestran que hay una brecha por maternidad del $51 \%$, pero al corregir por sesgo de selección esta aumenta a $65,9 \%$. Los instrumentos elegidos están correlacionados con otras variables de interés como la educación propia, y por ende los resultados tan extremos pueden ser producto de este sesgo. En línea con lo anterior, Olarte y Peña (2010) encontraron evidencia de que ser madre incrementa la probabilidad de ser informal. Concluyen que, controlando por estado civil y otras variables dependientes, al ser madre existe mayor posibilidad de ocuparse en oficios del hogar, siendo este efecto más notorio en las madres con hijos entre 0 y 5 años. Las autoras calculan que la brecha salarial bruta por maternidad es de alrededor de $17.6 \%$ para mujeres entre 18 y 65 años de edad. Corrigiendo el sesgo de selección y controlando factores observables como el capital humano, la estructura del hogar y la región, aún subyace un diferencial del $9.4 \%$, que se hace más pronunciado para mujeres con hijos en edades entre los 0 y los 5 años de edad.

Ramírez et. al. (2015) analizaron el efecto del aumento de la licencia de maternidad de 12 a 14 semanas en 2011 y encontraron que causó un deterioro en las condiciones de trabajo de las mujeres en edad fértil, en comparación con otros grupos de trabajadores. En términos absolutos, la probabilidad de participar en el mercado laboral disminuyó, tanto en el sector formal como en el informal de la economía. Los grupos de comparación en este trabajo están compuestos por mujeres en edad fértil, entre 18 y 30 años de edad, en comparación con el grupo de mujeres entre 40 y 55 años de edad, asociadas a bajas tasas de fecundidad y a 
aquellas que no están afectadas por la legislación, además de un análisis del grupo de hombres. Este último grupo es de gran interés, dado que tienen el mismo rango de edad, experiencia y educación, lo que permite hacer comparaciones. En este sentido, y como resultado de la extensión de la licencia, las mujeres tenían más probabilidades de estar desempleadas o ser informales, así como de recibir salarios más bajos en comparación con los hombres, incluso controlando por sus características observables.

A partir de la experiencia internacional, se presenta el modelo a estimar a partir de una comparación de los grupos afectados (mujeres fértiles) y no directamente afectados por la medida (mujeres en edad no fértil y hombres). El aporte del documento es el análisis no experimental, que aísla el efecto geográfico de los mercados laborales, al igual que de otros factores como la edad mínima para entrar al mercado laboral, la comparabilidad entre sectores económicos y los niveles educativos. Igualmente, al no afectar a los hombres, la Ley permite realizar mejores inferencias de los efectos sobre las tasas de ocupación e informalidad.

\section{METODOLOGÍA}

\section{A. Datos}

Los datos utilizados provienen de la Gran Encuesta Integrada de Hogares (GEIH) del Departamento Administrativo Nacional de Estadística (DANE) entre enero y septiembre de 2015 y 2017 en las capitales principales del país. La elección de los trabajadores del sector urbano se hace para intentar eliminar la dualidad del mercado laboral colombiano, donde el sector urbano tiene diferencias significativas con el rural, como por ejemplo la edad diferente para definir la población económicamente activa, la alta informalidad y las diferencias en los sectores económicos, entre otros factores. ${ }^{1}$ En este sentido, se estudió un total

${ }^{1}$ El criterio de informalidad es el mismo utilizado por el DANE. Según Quejada et. al. (2014) la definición de informalidad incluye los siguientes grupos de trabajadores: 1) Los empleados particulares y los obreros que laboran en establecimientos, negocios o empresas que ocupan hasta cinco personas en todas sus agencias y sucursales, incluyendo al patrono; 2) Trabajadores familiares sin remuneración; 3) Trabajadores sin remuneración en empresas o negocios de otros; 4) Empleados domésticos; 5) Trabajadores por cuenta propia, y 6) Patronos de empresas de hasta diez trabajadores. 
de 17,3 millones de trabajadores, aplicando los factores de expansión, en 24 departamentos del área urbana. Las mujeres representan el 45\% del total de la población ocupada y están sub-representadas en la población entre 20 y 30 años, como se aprecia en la pirámide de la población ocupada en Colombia en 2017 en el Gráfico 1.

Los datos de las encuestas de hogares muestran que la participación en el empleo de las mujeres que habitan en áreas urbanas ha aumentado en los últimos quince años en Colombia. Su tasa de participación ha subido de 53\% a 58\% en 2017; del mismo modo, su tasa de empleo ha aumentado del $42 \%$ al 50\%, en línea con la de los hombres.

\section{GRÁFICO 1}

Colombia: Pirámide de la población ocupada, 2017

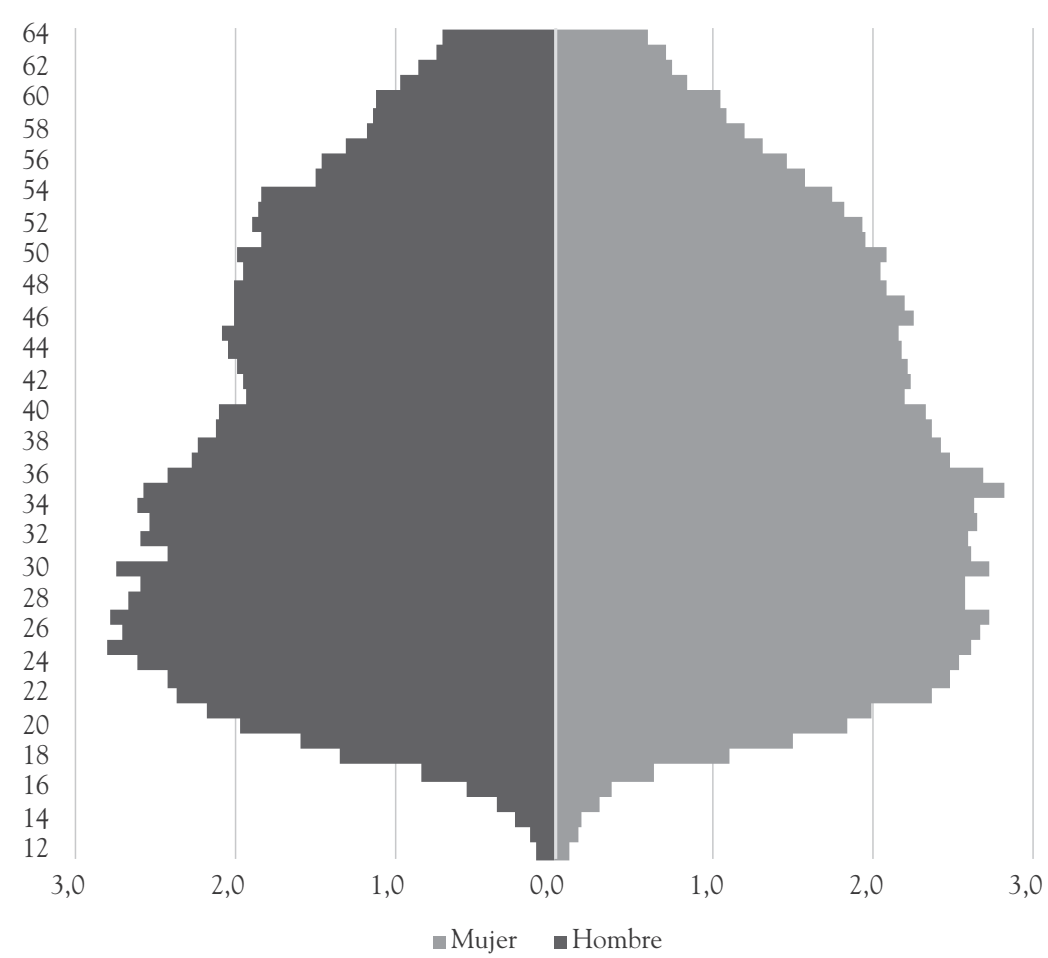

Fuente: DANE, Gran Encuesta Integrada de Hogares, 2015-2017. 


\section{GRÁFICO 2}

Colombia: Tasas generales de participación y tasas de ocupación por sexo, 2001, 2009 y 2017.

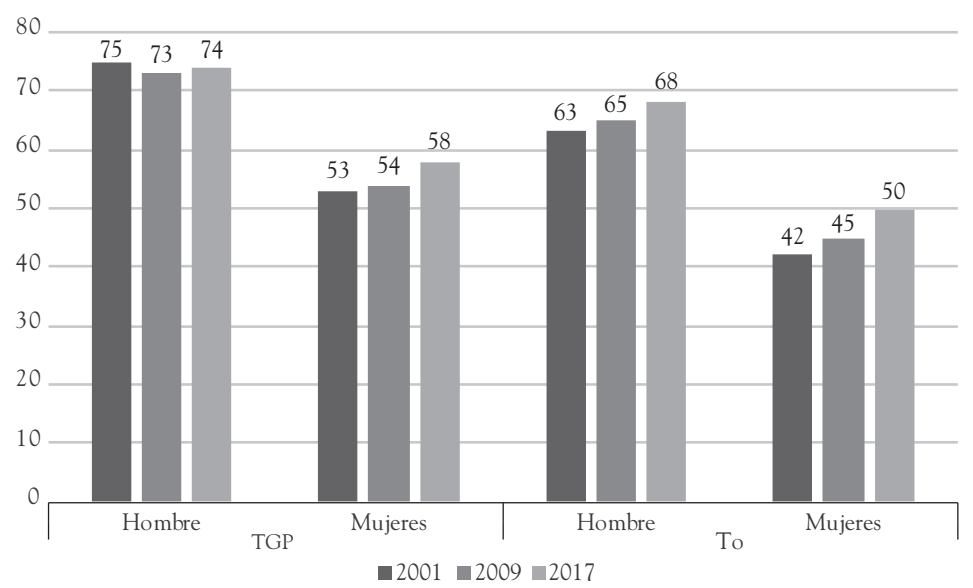

Fuente: DANE, Encuesta de Hogares, 2001-2017.

Luego se realizó una exploración estadística de los datos de las bases de datos recopiladas, que son importantes para la interpretación posterior de los resultados de estimaciones.

Se analizaron 326 mil hombres; el grupo en edad mayormente fértil fue de 92 mil y 192 mil para el grupo de mujeres mayores de 31 años. La edad mediana de los hombres fue de 37 años; para los grupos de mujeres fueron de 25 y 43 años, respectivamente. En términos de niveles de educación, las mujeres jóvenes se mostraron más educadas al estar ubicadas en los niveles superiores de educación frente a sus pares. En la distribución por relación a la cabeza del hogar, los hombres son en general cabezas de hogar (60\%) frente a las mujeres jóvenes (14\%) y las mayores a treinta años $(38 \%)$.

\section{B. Modelo econométrico}

Se estima un modelo econométrico para calcular las diferencias en las tasas de ocupación e informalidad entre los grupos de tratamiento y de control en 


\section{CUADRO 2}

Estadísticas descriptivas por grupos de análisis

\begin{tabular}{|l|l|r|r|r|}
\hline \multicolumn{1}{|c|}{ Variable } & \multicolumn{1}{|c|}{$\begin{array}{c}\text { Característica } \\
\text { evaluada }\end{array}$} & $\begin{array}{c}\text { Hombres } \\
15-65\end{array}$ & $\begin{array}{c}\text { Mujeres } \\
15-30\end{array}$ & \multicolumn{1}{c|}{$\begin{array}{c}\text { Mujeres } \\
40-65\end{array}$} \\
\hline \multirow{4}{*}{$\begin{array}{l}\text { Distribución por niveles } \\
\text { educativos }\end{array}$} & Primaria / ninguno & 23,12 & 6,46 & 27,52 \\
\cline { 2 - 5 } & Secundario $\left(6^{\circ}-9^{\circ}\right)$ & 17.74 & 11.46 & 16.51 \\
\cline { 2 - 5 } & Media $\left(10^{\circ}-13^{\circ}\right)$ & 32.70 & 37.48 & 26.71 \\
\cline { 2 - 5 } & Pregrado o superior & 26.44 & 44.60 & 29.26 \\
\hline Edad (mediana por grupo) & Cabeza de hogar & 59,89 & 14,35 & 38,47 \\
\hline \multirow{4}{*}{$\begin{array}{l}\text { Distribución como relación } \\
\text { de la cabeza de hogar }\end{array}$} & Pareja, cónyuge, & 4,6 & 24,33 & 40,32 \\
\cline { 2 - 5 } & Hijo, hijastro & 24.10 & 43.25 & 12.88 \\
\cline { 2 - 5 } & Nieto & 1,53 & 2,94 & 0,18 \\
\cline { 2 - 5 } & Otros parientes & 8,23 & 12,93 & 7,41 \\
\cline { 2 - 5 } & Servicio doméstico & 1,65 & 2,2 & 0,74 \\
\hline \multicolumn{2}{|l|}{ Total de personas en el grupo } & 326,909 & 92,504 & 192,074 \\
\hline
\end{tabular}

Fuente: DANE, Gran Encuesta Integrada de Hogares, 2017.

los períodos anteriores y posteriores a la medición (2017). En primer lugar, el fenómeno de la ocupación y la informalidad laboral, Y puede suscribirse a un fenómeno de probabilidad, donde uno (1) corresponde a estar en el estado y cero (0) en el caso opuesto. Asimismo, el cambio de la legislación sobre la licencia de maternidad ocurrido una sola vez en enero de 2017, está modelado por una variable dicotómica llamada L2017.

Los grupos de tratamiento son los siguientes: el grupo de mujeres de 18 a 30 años; el grupo de control, formado por mujeres de 40 a 55 años, y hombres de 18 a 65 años. Cada uno de ellos interactuará con la variable ficticia L2017 para estimar el efecto del aumento de la licencia de maternidad en los resultados del mercado laboral.

Existen diferentes especificaciones econométricas para evaluar el impacto de esta política en la población femenina. Sin embargo, las formas funcionales de la ecuación objetivo se pueden representar de la siguiente manera:

$$
Y=\beta_{o}+\beta_{1} X+\beta_{2} \operatorname{Exp} 2+\beta_{3} L 2017+\beta_{4} G+\beta_{5} L 2017 * G+\varepsilon
$$


Donde,

Y identifica la condición del individuo en informalidad u ocupación.

El impacto es la dimensión de los coeficientes, $\beta$, que captan la contribución marginal de cada factor a la probabilidad de empleo e informalidad. Específicamente, el término constante marca la tasa $\beta_{0}$ media de empleo y la informalidad de la población de la muestra).

La variable $G$ representa cada uno de los grupos etarios planteados para comprobar el efecto de la ley en su situación laboral. En este aspecto, cabe mencionar que la introducción de otras características se hace con el deseo de aislar otros factores que podrían afectar la ocupación laboral y la informalidad.

Se introduce un conjunto de variables $(\mathrm{X})$ que incluyen la edad, la edad al cuadrado, la educación, la ubicación, el parentesco dentro del hogar, el número de hijos, el número total de miembros, el mes de la encuesta y el estrato económico.

El método de estimación es un modelo Logit por máxima verosimilitud. Esto asegura la consistencia de los coeficientes después de maximizar la función de log-verosimilitud.

Para el caso particular de la informalidad, en Colombia se han realizado varios estudios de las variables que determinan este fenómeno, Calderón et. al. (2017), Gustavo Adolfo (2008) y Quejada Pérez et. al. (2014) incluyen entre sus estimaciones modelos de variables de elección discreta asociadas a la condición socioeconómica de los individuos, tales como la edad, el parentesco, la ubicación geográfica, los sectores sociales y económicos. Estas variables se introducen en el vector (X) utilizadas en este trabajo.

El primer indicador de eficiencia del modelo se calcula midiendo la varianza de la variable dependiente capturada por las variables de control. Mientras mayor sea este indicador, $\mathrm{R}^{2}$, más efectivo es el modelo para determinar el comportamiento de la variable dependiente. Junto a esto está el indicador de consistencia global del conjunto de variables, que debe ser significativo en un 5\% para rechazar la hipótesis nula. La tercera vía es la capacidad del modelo para clasificar correctamente las observaciones en grupos que correspondan a las características reales observadas de los individuos. Esto se hace tabulando el número de casos que fueron estimados como verdadero o falso y fueron clasificadas como tales, evitando las situaciones que se conocen como errores de hipótesis I y II, respectivamente. Todas las regresiones se realizan utilizando los factores de expansión en el software STATA 14. 


\section{Resultados}

El Cuadro 3 muestra los resultados de las estimaciones de la participación laboral y el Cuadro 4, de la informalidad. En términos de estimación global, los modelos son aceptables, considerando el valor de la estadística de chi2 global, que es significativa al 5\% (5.013). A su vez, todas las variables introducidas presentaron niveles de significación estadística del 5\% y según el $\mathrm{R}^{2} \mathrm{McFadden}$ el modelo consigue explicar el $18 \%$ y el $33 \%$, de la varianza. El porcentaje de casos correctamente clasificados dentro del modelo propuesto alcanzó alrededor del $72 \%$ y el $76 \%$, respectivamente.

Para la interpretación en el modelo probabilístico los coeficientes miden el efecto marginal según el tipo de variable. Para las continuas, este efecto es el porcentaje por el cual la probabilidad de estar en el mercado laboral o de ser informal aumenta cuando ocurre un cambio del $1 \%$ en la variable independiente. Para las categóricas, esta variación se mide en relación con la de una característica básica. Por ejemplo, la probabilidad de que una persona con educación primaria participe en el mercado laboral es de $10 \%$ más que en relación en las personas que no tienen ningún nivel educativo. Este resultado es consistente con los de trabajos que emplean metodologías similares, como Arango y Posada (2003) y Mora (2009).

Con la prueba de robustez del modelo, se examina cada uno de los coeficientes asociados a los diferentes grupos de población. Cabe mencionar que, en relación con el período 2015-16, la participación laboral se incrementó en un 2.8\% en 2017 para todos los grupos. En promedio, entre 2015 y 2016, las mujeres de 15 a 30 años y los hombres de 15 a 65 años tenían una tasa de participación laboral del 6,9\%, un 23,2\% más alta que las mujeres de 30 a 65 años. Cuando la variable dummy 2017 es interactuada con los mismos grupos de población, se observa que la tasa de participación laboral, controlada por múltiples características socioeconómicas, disminuyó $1.4 \%$ en las mujeres en edad fértil y aumentó para los hombres en 1.3\%, en comparación con las mujeres de 30 a 65 años de edad. Esta compensación entre el empleo femenino y el masculino se debe al mayor costo que la gente debe asumir por el aumento de la licencia de maternidad, resultado que está en línea con los resultados encontrados por Ramírez et. al. (2015), aunque vale la pena mencionar que la presente estimación es más limpia que la del mencionado estudio, ya que la reforma de 2017 no afectó la licencia de paternidad.

Los resultados de la informalidad muestran que, en 2017, esta se mantuvo estadísticamente estable en comparación con 2015-2016. Por grupos, las mujeres 


\section{CUADRO 3}

Estimación de la probabilidad de participar en el mercado laboral

\begin{tabular}{|c|c|c|c|c|c|c|}
\hline Variable & $\begin{array}{c}\text { Característica } \\
\text { evaluada }\end{array}$ & Coeficiente & $\begin{array}{c}\text { Efecto } \\
\text { Marginal }\end{array}$ & Error & $\mathrm{P}($ valor $)$ & $\begin{array}{c}\text { Característica } \\
\text { base }\end{array}$ \\
\hline Dummy ley & 2017 & 0.07 & $2.8 \%$ & 0.00 & 0.00 & $2015-2016$ \\
\hline \multirow[t]{2}{*}{ Dummy grupos } & Mujeres $15-30$ & 0.18 & $6.9 \%$ & 0.00 & 0.00 & M30-65 \\
\hline & Hombres $15-65$ & 0.61 & $23.2 \%$ & 0.00 & 0.00 & \\
\hline \multirow[t]{2}{*}{ Interacciones } & $2017^{*} \mathrm{M} 15-30$ & -0.04 & $-1.4 \%$ & 0.00 & 0.00 & $2017 * \mathrm{M} 30-65$ \\
\hline & $2017 * \mathrm{H} 15-65$ & 0.03 & $1.3 \%$ & 0.00 & 0.00 & \\
\hline \multirow[t]{4}{*}{ Educación } & Primaria $\left(1^{\circ}-50\right)$ & 0.27 & $10.3 \%$ & 0.00 & 0.00 & \multirow[t]{4}{*}{ Ninguno } \\
\hline & Secundario $\left(6^{\circ}-9^{\circ}\right)$ & 0.35 & $13.1 \%$ & 0.00 & 0.00 & \\
\hline & $\operatorname{Media}\left(10^{\circ}-13^{\circ}\right)$ & 0.71 & $25.9 \%$ & 0.00 & 0.00 & \\
\hline & Pregrado & 0.82 & $30.0 \%$ & 0.00 & 0.00 & \\
\hline Edad $^{2}$ & Continua & -0.44 & $-17.1 \%$ & 0.00 & 0.00 & Continuar \\
\hline \multirow{5}{*}{$\begin{array}{l}\text { Relación cabeza } \\
\text { de hogar }\end{array}$} & Pareja, cónyuge, & -0.29 & $-11.5 \%$ & 0.00 & 0.00 & \multirow[t]{5}{*}{ Jefe de hogar } \\
\hline & Hijo, hijastro & -0.54 & $-21.1 \%$ & 0.00 & 0.00 & \\
\hline & Nieto & -0.80 & $-30.7 \%$ & 0.00 & 0.00 & \\
\hline & Otros parientes & -0.41 & $-16.2 \%$ & 0.00 & 0.00 & \\
\hline & Servicio doméstico & 1.44 & $36.7 \%$ & 0.00 & 0.00 & \\
\hline \multirow[t]{4}{*}{ Hijos } & 1 & 0.12 & $4.6 \%$ & 0.00 & 0.00 & \multirow[t]{5}{*}{ Ninguno } \\
\hline & 2 & 0.11 & $4.2 \%$ & 0.00 & 0.00 & \\
\hline & 3 & 0.11 & $4.3 \%$ & 0.00 & 0.00 & \\
\hline & 4 o mas & 0.16 & $6.2 \%$ & 0.00 & 0.00 & \\
\hline \multicolumn{2}{|l|}{ Constante } & -3.48 & $2.2 \%$ & -161.65 & 0.00 & \\
\hline $\mathrm{R}^{2}$ & 0.1782 & & & & & \\
\hline Observaciones & $1,160,115$ & & & & & \\
\hline
\end{tabular}

Fuente: Cálculos de los autores basados en DANE, Gran Encuesta Integrada de Hogares, 2015 - 2017.

de 15 a 30 años y los hombres de todas las edades tenían tasas de informalidad más bajas que las mujeres mayores de 30 años, exactamente $2 \%$ y $5 \%$, respectivamente. En relación a la hipótesis de este trabajo, la implementación de la nueva ley de maternidad incrementó en un 0,57\% la probabilidad de informalidad de las mujeres de 15 a 30 años en comparación con los otros grupos. Este aumento porcentual también se presentó en 2014, como lo demuestran Ramírez et. al. (2015). 


\section{CUADRO 4}

Estimación de la probabilidad de que sea informal

\begin{tabular}{|c|c|c|c|c|c|c|}
\hline Variable & $\begin{array}{c}\text { Característica } \\
\text { evaluada }\end{array}$ & Coeficiente & $\begin{array}{c}\text { Efecto } \\
\text { Marginal }\end{array}$ & Error & $\mathrm{P}($ valor $)$ & $\begin{array}{c}\text { Característica } \\
\text { base }\end{array}$ \\
\hline \multirow{2}{*}{$\begin{array}{l}\text { Dummy } \\
\text { grupos }\end{array}$} & Mujeres 15-30 & -0.06 & $-2.35 \%$ & 0.00 & 0 & \multirow[t]{2}{*}{ M30-65 } \\
\hline & Hombres $15-65$ & -0.14 & $-5.22 \%$ & 0.00 & 0 & \\
\hline Interacciones & $2017^{*} \mathrm{M} 15-30$ & 0.01 & $0.57 \%$ & 0.00 & 0.005 & $2017^{*} \mathrm{M} 30-65$ \\
\hline \multirow{6}{*}{$\begin{array}{l}\text { Relación } \\
\text { cabeza de } \\
\text { hogar }\end{array}$} & Pareja, cónyuge. & 0.11 & $4.32 \%$ & 0.00 & 0 & \multirow[t]{6}{*}{ Jefe de hogar } \\
\hline & $2017 * \mathrm{H} 15-65$ & 0.03 & $1.3 \%$ & 0.00 & 0.00 & \\
\hline & Hijo, hijastro. & 0.16 & $6.15 \%$ & 0.00 & 0 & \\
\hline & Nieto. & 0.27 & $9.82 \%$ & 0.01 & 0 & \\
\hline & Otros parientes. & 0.03 & $1.34 \%$ & 0.00 & 0 & \\
\hline & Servicio doméstico. & 0.28 & $10.14 \%$ & 0.05 & 0.052 & \\
\hline \multirow[t]{4}{*}{ Educación } & Primaria $\left(1^{\circ}-5^{\circ}\right)$ & -0.28 & $-11.09 \%$ & 0.01 & 0 & \multirow[t]{4}{*}{ Ninguno } \\
\hline & Secundario $\left(6^{\circ}-9^{\circ}\right)$ & -0.37 & $-14.72 \%$ & 0.01 & 0 & \\
\hline & Media $\left(10^{\circ}-13^{\circ}\right)$ & -0.85 & $-32.73 \%$ & 0.01 & 0 & \\
\hline & Pregrado & -1.19 & $-44.79 \%$ & 0.01 & 0 & \\
\hline \multirow[t]{3}{*}{ Hijos } & 1 & -0.08 & $-2.96 \%$ & 0.00 & 0 & \multirow[t]{3}{*}{ Ninguno } \\
\hline & 2 & -0.08 & $-3.27 \%$ & 0.00 & 0 & \\
\hline & 3 & -0.06 & $-2.30 \%$ & 0.00 & 0 & \\
\hline $\mathrm{Edad}^{2}$ & Continua & -0.19 & $-7.16 \%$ & 0.00 & 0 & Continua \\
\hline \multicolumn{2}{|l|}{ Constante } & -0.76 & $3.51 \%$ & 0.00 & 0 & \\
\hline $\mathrm{R}^{2}$ & 0.3335 & & & & & \\
\hline Observaciones & 668.118 & & & & & \\
\hline
\end{tabular}

Fuente: Cálculos de los autores basados en DANE, Gran Encuesta Integrada de Hogares, 2015 - 2017.

Además, las pruebas estadísticas muestran que la tasa de informalidad de los hombres no se vio afectada.

Entre otros resultados de interés, se encuentra que las personas con un mayor nivel de educación tienen menores tasas de informalidad, también como jefes de hogar. También se encuentra que los sectores económicos como el comercio son los que tienen más probabilidades de tener empleados informales, hallazgos que están en línea con trabajos como el de Calderón et. al. (2017), 


\section{CONCLUSIONES}

Los resultados indican que, debido a la extensión de cuatro semanas en el período de licencia por la nueva ley de maternidad en Colombia, las mujeres entre 18 y 30 años tienen más probabilidades de no participar en el mercado laboral o de ser empleadas en la informalidad. Los resultados fueron robustos y estuvieron en línea con lo encontrado en la literatura internacional asociada a los determinantes de la participación y la informalidad de las mujeres. En este orden de ideas, el mercado laboral colombiano se ve afectado negativamente por el aumento de las semanas de licencia de maternidad, obteniendo resultados contrarios a los deseados por los responsables de la política pública. En la literatura se ha propuesto la posibilidad de eliminar este sesgo en contra de las mujeres al igualar la licencia de paternidad con la licencia de maternidad, de manera que los empleadores no tendrían incentivos para discriminar. Según varios estudios, la ampliación de la licencia de paternidad también tiene otros beneficios, ya que proporciona un papel más activo en la crianza de los hijos que benefician al niño y reduce el estrés de la madre, mejorando su salud y la del recién nacido.

La investigación es consciente de que existe un conjunto de variables inobservables que pueden estar afectando la probabilidad de participación o de ser informales, especialmente desde el punto de la demanda laboral. Además, este trabajo sólo ha controlado las variables disponibles en la base de datos de las encuestas de hogares en Colombia y no usa experimentos para medir el sesgo en la contratación. Los resultados de este trabajo no son comparables con los de otros países, dadas las grandes diferencias entre los distintos regímenes laborales. En futuros trabajos se puede considerar el uso de estas nuevas técnicas para evaluar la percepción cultural que tienen las mujeres y su licencia de maternidad en la contratación. Igualmente, al intentar usar datos longitudinales se pueden aplicar técnicas de evaluación de impacto más acordes con la evaluación buscada.

\section{REFERENCIAS}

Addati, L., N. Cassirer and K. Gilchrist (2014), Maternity and paternity at work: Law and practice across the world, International Labour Office.

Arango, L. E., y C. E. Posada (2003), La participación laboral en Colombia, Fedesarrollo. Bogota. 
Baker, M., and K. Milligan (2008), "Maternal Employment, breastfeeding, and health: Evidence from maternity leave mandates", Journal of Health Economics, 27.

Baum, C. (2003), "The Effect of State Maternity Leave Legislation and the 1993 Family and Medical Leave Act on Employment and Wages", Labor Economics, 10.

Bergemann, A., and R. T. Riphahn (2010), "Female labour supply and parental leave benefits-the causal effect of paying higher transfers for a shorter period of time", Applied Economics - Letters, 18(1).

Blau, F. D., and L. M. Kahn (2013), "Female labor supply: Why is the US falling behind?", No. w18702, National Bureau of Economic Research.

Botello, H. A., y A. L. Alba (2014), "El efecto de la maternidad sobre los salarios femeninos en Latinoamérica”, Semestre Económico, 17(36).

Botello Peñaloza, H. A. (2015), "Empoderamiento de la mujer latinoamericana: empleo y educación, 1960-2010”, Tendencias E Retos, 20(2).

Botello-Peñaloza, H. A., \& Guerrero-Rincón, I. (2017). Condiciones para el empoderamiento de la mujer rural en Colombia. Entramado, 13(1), 62-70.

Calderón, A. N. C., Hernández, L. Y. C., \& Cañas, I. E. (2017). Determinantes socioeconómicos de la informalidad laboral y el subempleo en el área metropolitana de Bucaramanga, Colombia. Equidad y Desarrollo, (29), 53-82.

Correll, S. J., S. Benard and I. Paik (2007), "Getting a job: Is there a motherhood penalty? American Journal of Sociology, 112(5).

Cuddy, A. J., S. T. Fiske and P. Glick (2004), "When professionals become mothers, warmth doesn't cut the ice”, Journal of Social Issues, 60(4).

De Cabo, G., y M. Rodríguez (2014), "Elaboración de un índice sintético de discriminación salarial”, Madrid: Centro de Estudios Económicos Tomillo.

Eagly, A., and L. L. Carli (2007), Through the Labyrinth: The Truth about How Women Become Leaders, Harvard Business Press.

Espino, Alma, y Soledad Salvador (2014), "Un análisis de género de los costos laborales en Colombia”, Consultoría para el Ministerio del Trabajo de Colombia, Mimeo.

Forero de Saade, María Teresa, Leonardo Cañón Ortegón y Javier Armando Pineda Duque (1991), Participación de la mujer en el trabajo. Mujer trabajadora. Nuevo compromiso social, Estudios Sociales Juan Pablo II.

Goldin, Claudia (2014), "A Grand Gender Convergence: Its Last Chapter", American Economic Review, 104(4). 
Gruber, J. (1994), "The Incidence of Mandated Maternity Benefits, The American Economic Review, 84(3)

Gutiérrez, Diego (2008), "Efectos de la fecundidad sobre el ingreso laboral femenino", Tesis de Maestría, Facultad de Economía, Universidad de los Andes, Bogotá.

Gustavo Adolfo, G. C. (2008), "Informalidad regional en Colombia. Evidencia y determinantes” Revista Desarrollo y Sociedad, (61).

Hashimoto, M., R. Percy, T. Schoellner and B. A. Weinberg (2004), "The long and short of it: maternity leave coverage and women's labor market outcomes", IZA Discussion Papers, No. 1207, Institute of Labor Economics, Bonn.

Hyclak, T., G. Jones and R. Thornton (2012), Fundamentals of labor economics, Mason, Ohio: South-Western, Cengage Learning

Iragorri, C. M. (2012), "La ley de protección a la maternidad como incentivo de participación laboral femenina: El caso colombiano”, Coyuntura Económica.

Jaumotte, F. (2003), Female Labour Force Participation: Past Trends and Main Determinants in OECD Countries (No. 376), OECD Publishing.

Lagarde C. (2014), Atreverse a aprovechar la diferencia: Las tres claves para el empoderamiento de la mujer, Washington,D. C., Fondo Monetario Internacional, Ponencia en el National Democratic Institute, 19 de mayo

Lai, Y. C., and S. Masters (2005), "The effects of mandatory maternity and pregnancy benefits on women's wages and employment in Taiwan, 1984-1996”, ILR Review, 58(2), 274-281.

Livermore, Tanya, Joan Rodgers and Peter Siminski (2011). "The Effect of Motherhood on Wages and Wage Growth: Evidence for Australia”, Economic Record, 87(1).

Martin, R, A. Ness, D. Gunnell, P. Emmet and G. Davey, ALspac Study Team (2004), "Does breast-feeding in infancy lower blood pressure in childhood?", The Avon Longitudinal Study of Parents and Children (ALSPAC), Circulation, 16:109.

Mora, J. J. (2009), "La relación entre la participación laboral y las remesas en Colombia”, Documentos de Trabajo, No. 005246, Universidad Icesi, Cali.

Olarte, L., y X. Peña X. (2010), "El efecto de la maternidad sobre los salarios femeninos”, Documento CEDE No. 2010-18, Facultad de Economía, Universidad de los Andes, Bogotá.

Oaxaca, R. L. (2007), "The challenge of measuring labor market discrimination against women” Swedish Economic Policy Review, 14(1). 
Quejada Pérez, R., M. Yánez Contreras, M., y K. Cano Hernández (2014), "Determinantes de la informalidad laboral: Un análisis para Colombia”, Investigación y Desarrollo, 22(1).

Ramírez, N., A. M. Tribin Uribe, and C. Vargas (2015), "Maternity and Labor Markets: Impact of Legislation in Colombia", Inter-American Development Bank, Washington.

Rossin-Slater, M., C. J. Ruhm, and J. Waldfogel (2013), "The Effects of California's Paid Family Leave Program on Mothers' Leave-Taking and Subsequent Labor Market Outcomes, Journal of Policy Analysis and Management, 32(2).

Schönberg, U., and J. Ludsteck (2007), "Maternity Leave Legislation, Female Labor Supply, and the Family Wage Gap." IzA Discussion Papers, No. 1699, [http://nbnresolving.de/urn:nbn:de:101:1-200804110188]

Victora, C, F. Barros, F. Lima, B. Horta and J. Wells (2003), "Anthropometry and body composition of 18 year old men according to duration of breast-feeding: Birth cohort study from Brazil”, BMJ, 18:327-901. 